\title{
A Clinical Study of Pitutary and Pineal Gland Interactions: Effects of Treatment with The Pineal Hormone Melatonin in Patients with Hypophyseal Adenomas
}

\author{
Lissoni P, Pasquetti M, Pensato S, Messina G, Porro G, Mainini E*, Brivio E, Di Fede G \\ Institute of Biological Medicine \\ *Endocrinology Division, Gallarate Hospital, Gallarate, Milan, Italy
}

*Address for Correspondence: Paolo Lissoni, E mail: paolo.lissoni@gmx.com

Received: 11 September 2019; Accepted: 22 November 2019; Published: 27 November 2019

Citation of this article: Lissoni P, Pasquetti M, Pensato S, Messina G, Porro G, et al. (2019) A Clinical Study of Pituitary and Pineal Gland Interactions: Effects of Treatment with The Pineal Hormone Melatonin in Patients with Hypophyseal Adenomas. Rea Int Journal of Endocrinology and Diabetes. 1(1): 006-010. DOI: 10.37179/rijed.000002.

Copyright: (C) 2019 Lissoni P, et al. This is an open access article distributed under the Creative Commons Attribution License, which permits unrestricted use, distribution, and reproduction in any medium, provided the original work is properly cited.

\begin{abstract}
Despite the well demonstrated role of both pituitary and pineal glands in the regulation of the endocrine system, the knowledge of the functional relation between hypothesis and epiphysis is one of the less established aspect of the human physiology, particularly in the case of pituitary tumors, even though the inhibitory effect of the pineal on tumor cell proliferation has been confirmed by several experimental conditions.

The present study was carried out to study the effects of a chronic administration of the most investigated pineal hormone, melatonin (MLT) in patients with hypophyseal tumors, which did not respond to the conventional therapies. The study included 14 patients (prolactinoma:6; acromegaly:5; ACTH secreting tumor:2; chromophore tumour:1), who were compared with a control group of 50 healthy subjects.

The pineal function was evaluated by measuring the daily and nightly urinary excretion of the mail MLT metabolite, the sulfatoxymelatonin (6-MTS). Patients affected by pituitary tumors showed significantly higher mean values of 6-MTS during the light phase of the day than controls, whereas no difference was found in the night mean values. After an oral administration of MLT at a pharmacological dose of $20 \mathrm{mg} / \mathrm{day}$ in the late evening for 3 consecutive months, GH and PRL mean values decreased on MLT therapy in acromegaly and prolactinoma patients, respectively, without, however, significant differences. Moreover, a rapid normalization of ACTH levels was achieved in one of the two patients suffering from ACTH secreting tumor. Hypophyseal tumor dimension remained substantially unchanged on study.

These preliminary results would suggest an enhanced pineal function with loss of its physiological circadian rhythmicity in patients suffering from pituitary tumors and that the pineal hormone may potentially display an inhibitory effect on pituitary tumor cell proliferation, including patients with pituitary tumors for whom no other effective conventional therapy may be available.
\end{abstract}

Keywords: Melatonin, Pineal gland, Pituitary tumors

\section{Introduction}

Until about 50 years ago, the pineal gland, also called epiphysis, was taken into consideration as important for the human life by the only philosophic tradition as the potential site of the soul, whereas it was excluded from the clinical investigations by also the same Endocrinologists, despite its endocrine nature.

The pineal gland was progressively considered as potentially important also from a clinical point of view after the discovery of its endocrine activity, namely consisting of the production of the indole hormone melatonin (MLT) [1] and its fundamental role as neurochemical transducer able to modulate the biological functions according to the universal information's, mainly the light/dark circadian rhythm, with a higher production of MLT during the night and a lower secretion during the light phase of the day [2].

However, MLT is not the only hormone produced by the pineal 
gland, since it produces at least three other indole hormones, mainly released during the light phase of the daily period and consisting of 5methoxytryptamine (5-MTT), 5-methoxytryptophol and 5-methoxyindole acetic acid, some peptides such as arginine vasotocin (AVT), and several other hormones of beta-carbolic nature, the most known of them is the 5-methoxy-1,2,3,4-tetrahydro-beta-carboline, also called pin line of pine line (PNL) [3,4].

Because of the fundamental role of the pineal gland in the neuroendocrine modulation of the biological functions, including endocrine, nervous, immune and cardiovascular activities [5], it would be important to evaluate the effects of the pineal gland, or of at least its most investigated hormone MLT, on the other structures involved in the neuroendocrine regulation of the different biological systems, mainly the endocrine system, and in particular its influence on the pituitary gland, which represents the master endocrine gland according to the common opinion of the Endocrinologists.

On the contrary, every though the existence of a fundamental regulatory connection between pituitary and pineal glands had probably been already understood at the beginning of the medical Sciences in the old Greece, as suggested by the same names of epiphysis for the pineal gland and hypothesis for the pituitary gland to put into evidence a regulation upon or under, respectively, the biological dynamics, the experimental data concerning the possible fundamental relationship between hypophyseal and pineal endocrine functions are limited and controversial, since MLT has been proven either to stimulate, to inhibit or to have no effect on the endocrine secretions of the anterior hypothesis [6-8] and very few clinical studies have been carried out up to now to explore the influence of the pineal gland on the pituitary endocrine function [9-11].

The well documented Antiproliferative activity of MLT, as well as of 5-MTT and PNL [3-4,12], would further justify the investigation of the pineal-hypophyseal interactions, particularly in the case of pituitary tumors.

In more detail, MLT may either inhibit or stimulate cell proliferation through a regulation of the apoptotic processes, by promoting the apoptosis in the case of rapidly proliferating cells and by inhibiting the apoptosis when it is excessively expressed.

At present, however, most experimental and clinical studies are limited to the influence of the pineal gland on prolactin (PRL) or growth hormone $(\mathrm{GH})$-secreting pituitary tumors, whereas few data only are available on adreno-corticotropin hormone (ACTH)secreting tumors. In any case, the precise relationship between MLT and the pituitary functions remains controversial, and it would change in relation to the evolution of the different living species. MLT has appeared to stimulate GH and PRL secretion in cultured pituitary cells, whereas no effect was seen on the other pituitary endocrine secretions [13].

MLT, as well as other pineal indoles, may exert an important ant gonadotropic activity in animal species, whereas it does not seem to inhibit the gonadal functions in humans [14]. As far as the pineal influence of pituitary tumors, the experimental data available in the literature have shown an inhibitory role of MLT on the growth of pituitary tumors, particularly on prolactinoma $[15,16]$.

The same somatostatin-analogues would inhibit GH secretion from GH-secreting tumors at least in part by stimulating MLT secretion [17]. The anti-proliferative activity of MLT on the pituitary adenomas is also suggested and confirmed by the experimental evidence that patellectomy may enhance the frequency of spontaneous pituitary tumors, whereas the administration of pineal extracts has been proven to inhibit pituitary cell proliferation $[18,19]$.

Moreover, as far as the pineal function in GH- and PRL-secreting pituitary tumors is concerned, the few available clinical studies are in agreement with the evidence of a compensatory enhanced production of MLT during the day with a following progressive disappearance of its physiological light/dark circadian rhythm [17,20-21], whereas normal MLT values have been described in ACTH secreting pituitary tumors [21].

Finally, the administration of somatostatin analogues has been proven to restore a normal light/dark MLT rhythm in acromegaly patients [17].

The acute administration of mild pharmacological doses of MLT would not influence GH and PRL secretion in GH- and PRL-secreting tumors, respectively [20], while there is no study on the effects of a chronic MLT administration in patients with pituitary tumors. At the other side, the eventual effects of the pituitary hormones on the pineal function, or at least on MLT secretion, are yet less investigated and established, even though the few experimental studies available up to now would suggest a potential stimulatory role of the pituitary gland on the pineal, since the hypophysectomy has been proven to induce histological alterations and atrophy of the pineal gland [22,23].

In any case, at present it has been shown that the responsiveness of the pituitary gland to MLT would be depending on animal species, dosage, time of administration and pubertal development state [24], and in particular it has been shown that an acute administration of MLT may inhibit GH and PRL secretions in the only prepubertal subjects, and stimulate PRL secretion in the only pubertal subjects, without any particular effect on the other hypophyseal hormones.

The controversial results reported in the literature about the influence of the pineal function on the pituitary gland would mainly be due to the fact that at least for MLT it has been demonstrated that its action on the pituitary functions would be the end result of three major mechanisms, consisting of a direct action of MLT on the pituitary cells, a regulation of the hypothalamic function, and a modulation of the neurotransmitter pathways involved in the control of the hypothalamus, in particular by increasing serotonin brain content [25].

The intra-pituitary production of cytokines, which may influence the hypophyseal hormone secretions, would also be under a pineal regulation, because of the fundamental role of the pineal gland in the control of the cytokine network [26].

Within the possible mechanisms involved in the influence of the pineal on the hypophysis, it is in particular the direct action of MLT on the pituitary gland to change in relation to several variables, namely time of the administration and sexual development, depending on the expression of MLT receptor (MT-R) at hypophyseal sites [23, 27]. According to the down-regulation mechanism, the hypophyseal expression of MT-R depends on MLT blood concentrations.

In the presence of high blood levels of MLT, as occurring during the night with respect to the light phase of the day and in the prepubertal phase of the life with respect to the post pubertal one 
Citation: Lissoni P, Pasquetti M, Pensato S, Messina G, Porro G, et al. (2019) A Clinical Study of Pituitary and Pineal Gland Interactions: Effects of Treatment with The Pineal Hormone Melatonin in Patients with Hypophyseal Adenomas. Rea Int Journal of Endocrinology and Diabetes. 1(1): 006-010. DOI: 10.37179/rijed.000002.

[24], the down-regulation mechanisms would allow a diminished expression of MT-R by the pituitary gland. Therefore, the hypophysis would be less responsive to the pineal influence or at least to MLT action in the prepubertal than in the postpubertal period of the light and early in the morning than in the late afternoon or in the evening. Finally, the pineal gland may express both opioid [28] and cannabinoid receptors [29].

Then, the pineal could exert its central neuroendocrine regulatory role by modulating the influence of both brain opioid and cannabinoid systems, which play a fundamental role in the control of both neurotransmission pathways and the hypothalamic-pituitary axis. This study was performed to evaluate the pineal function in patients suffering from pituitary tumors and the effect of a chronic MLT treatment on the endocrine secretion of the hypophyseal tumors.

\section{Patients and Methods}

The study included 14 patients (M/E: 6/8; median age: 44 years, range 28-72), who were affected by pituitary tumors, and had progressed under the conventional surgical, radio therapeutic or endocrine treatments. Eligibility criteria were, as follows: histologically proven pituitary adenoma, measurable lesions, lack of response to the previous conventional therapies, and no chronic therapy with drugs influencing the endocrine secretions.

Tumor histotypes were, as follows: prolactinoma: 6; acromegaly:5; ACTH-secreting adenoma:2; chromophore tumor:1. Each patient was evaluated for the pineal function and for the response to MLT therapy at pharmacological doses for 3 months. The clinical protocol was approved by the Ethical Committee, explained to each patient, and written consent was obtained.

The pineal function was investigated by measuring the daily ( 8 AM- $8 \mathrm{PM}$ ) and the night (8 PM-8 AM) urinary excretion of the main metabolite of MLT, the 6-suifatoxy-melatonin (6-MTS). 6-MTS levels were measured by an enzyme immunoassay (ELISA) method using commercially available kits. GH, PRL and ACTH were also measured by the ELISA method. Normal values of 6-MTS observed in our laboratory ( $95 \%$ confidence limits) were ranging from 0.4 to $2.2 \mathrm{mcg} / \mathrm{h}$ for the light period and from 0.9 to $5.6 \mathrm{mcg} / \mathrm{h}$ for the night one, and the circadian rhythm of the pineal function was considered to be within the normal range when the night values of 6-MTS were at least two times greater than those found during the light phase of the day.

The control group consisted of 50 age and sex-matched healthy subjects. Tumor dimension was measured by the brain NMR. After the evaluation of their pineal function, patients were treated with MLT at an oral pharmacological dose of $20 \mathrm{mg}$ once / day in the late evening, generally 30 minutes before sleeping, without interruption for 3 consecutive months. Hormonal and radiological before and after 3 months of MLT therapy. Data were reported as mean $+/-$ SE, and statistically analyzed by the Student's t test, the chi-square test and the analysis of variance, as appropriate.

\section{Results}

6-MTS urinary mean levels observed in patients and in control group are reported in Figure 1. Abnormally high daily values of 6-MTS were seen in 8/14 (57\%) patients (prolactinoma: 4; GHsecreting tumor: 3; ACTH secreting tumor:1). A lack of pineal

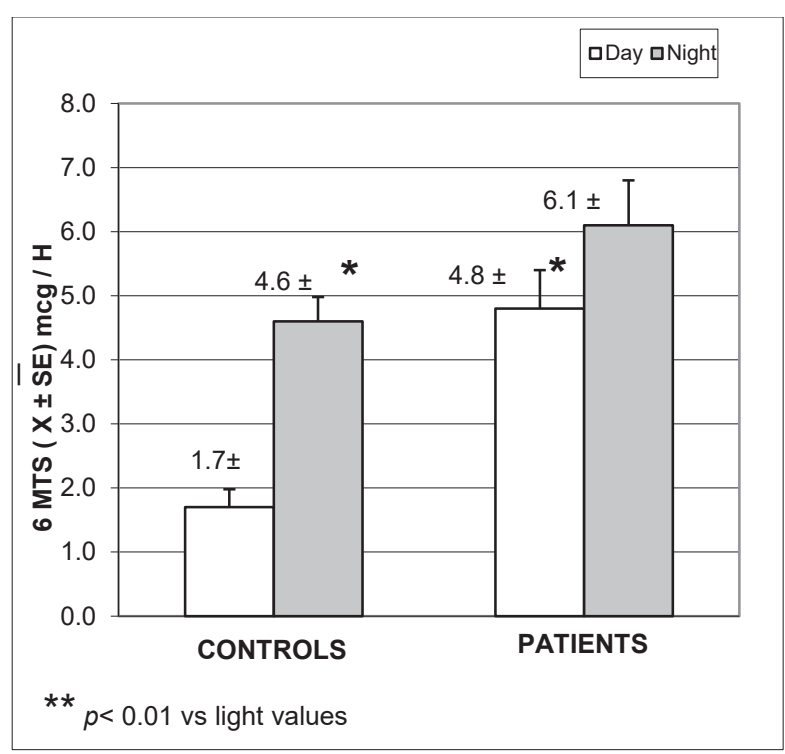

Figure 1: Day and night urinary mean values of 6-sulfatoxymelatonin (6MTS) in healthy controls and in patients with pituitary tumors.

circadian rhythm, with night values of 6-MTS less than at least two times greater than the daily ones was found in 10/14 (71\%) patients, and mean night values of 6-MTS found in patients were not significantly higher than those seen during the light phase of the day.

Moreover, daily mean values of 6-MTS observed in patients were significantly higher than in controls $(\mathrm{P}<0.01)$. Night mean values of 6-MTS were also higher in patients than in controls, but this difference was not statistically significant. A normalization of PRL levels was achieved in 1/6 (17\%) prolactinoma patients, and in none of the patients suffering from acromegaly. Moreover, a rapid normalization of ACTH levels, from 378 to $37 \mathrm{pg} / \mathrm{ml}$, was obtained

in one of the two patients affected by ACTH-secreting tumor. Both PRL and GH mean levels decreased under MLT therapy, without, however, significant differences with respect to the pretreatment concentrations (PRL: $46+1-8$ vs $65+1-9 \mathrm{ng} / \mathrm{ml}$; GH: $11+1-4$ vs $17+1-5 \mathrm{ng} / \mathrm{ml}$ ). MLT therapy was well tolerated by all patients, and most of them experienced relaxation, and no biological toxicity occurred. Finally, tumor dimensions remained substantially unchanged, and no further increase was seen on study.

\section{Conclusion}

In agreement with previous preliminary clinical observations, this study confirms that the presence of pituitary tumors is characterized by an altered pineal function consisting of an enhanced MLT production, but associated with a disappearance of the physiological light/dark circadian rhythm $[20,21]$. With respect to previous data reported by other authors [21], ACTH-secreting adenoma may be also characterized by an altered MLT secretion.

Moreover, the results of this preliminary study, which will have to be confirmed in a greater number of patients followed for a longer period of time, would seem to suggest that the pineal hormone at mild pharmacological doses may control the growth of the pituitary tumor cells and inhibit their endocrine secretion. Because of the absolute lack of biological toxicity and the dose-dependency of the 
Citation: Lissoni P, Pasquetti M, Pensato S, Messina G, Porro G, et al. (2019) A Clinical Study of Pituitary and Pineal Gland Interactions: Effects of Treatment with The Pineal Hormone Melatonin in Patients with Hypophyseal Adenomas. Rea Int Journal of Endocrinology and Diabetes. 1(1): 006-010. DOI: 10.37179/rijed.000002.

Antiproliferative properties of the pineal hormone [30], further promising results could be achieved by high-dose MLT in the treatment of pituitary tumors, for whom no other conventional therapy may be available.

Moreover, a longer period of MLT therapy could also allow a decrease in tumor dimensions of the pituitary adenomas. The mechanisms responsible for the enhanced MLT production in the presence of pituitary tumors need to be further investigated and understood; however, they could simply be the expression of a compensatory mechanism to control the growth of the pituitary tumor. From this point of view, the exogenous administration of MLT at pharmacological doses would amplify the same biological response of patients, consisting of an enhanced MLT production on the basis of its Antiproliferative activity $[4,7,12]$.

On the other hand, pituitary tumor-related pineal hyper function could simply represent the consequence of a possible stimulatory effect of the pituitary hormones on the pineal endocrine activity, as suggested by the experimental evidence of a pineal atrophy in hypophysectomized animals [22,23].

In any case, further studies, by investigating the other pituitary and hypothalamic endocrine secretions, as well as the functional status of other fundamental brain structures involved in the control of the hypothalamic pituitary axis, such as brain opioid [28] and cannabinoid systems [29], will be required to better define the mechanisms responsible for the enhanced MLT production in patients with pituitary tumors, as well as to explain the potential therapeutic effects of MLT in the treatment of the neoplastic disease of the pituitary gland.

In particular, it has to be established whether the potential anticancer properties of MLT against pituitary tumor growth may be the expression of the general anticancer activity of MLT [31,32], or whether it may depend on more specific mechanisms. Finally, at least two other pineal molecules, including 5-MTT and PNL [33], could also display antiproliferative properties on the pituitary tumors $[3,4]$.

Obviously, the too low number of patients and the different tumor histotypes does not allow to achieve define conclusion about the possible use of MLT in the treatment of pituitary tumors, but on the same time the results of this study may be sufficient to justify further clinical studies, not only to treat the pituitary tumors, but also to investigate the pineal-pituitary functional axis, which could exert a fundamental role in the regulation of the whole endocrine system in relation to the different living species $[2,27,34]$.

\section{References}

1. Wurtman RI, Axelrod I, Phillips LS (1963) Melatonin Synthesis in the pineal gland: control by light. Science 142: 1071-1073.

2. Axelrod I (1974) The pineal gland: a neurochemical transducer. Science 184: 1341-1348.

3. Sheliard SA, Wheian RDH, Hill BT (1989) Growth inhibitory and cytotoxic effects of melatonin and its metabolites on human tumour cell lines in vitro. Br J Cancer 60: 288-290.

4. Sze SF, Ng TB, Liu WK (1993) Antiproliferative effect of pineal indoles on cultured tumor cell lines. J Pineal Res 14: 27-33.

5. Wetterberg L (1978) Melatonin in humans Physiological and clinical studies. J Neural Transm Suppl 13: 289-310.

6. Tamarkin L, Westrom WK, Hamilt AI, Goldman BD (1976) Effects of melatonin on the reproductive system of male and female Syrian hamsters. A diurnal rhythm in sensitivity to melatonin. Endocrinology 99: 1534-1541.

7. Lapin V, Ebels I (1981) The role of the pineal gland in neuroendocrine control mechanisms of neoplastic growth. i Neural Transm 50: 275282.

8. Leadem CA, Blask DE (1981) Evidence for an inhibitory influence f the pineal gland on prolactin in the female rat. Neuroendocrinology 33: 268-275.

9. Smythe GA, Lazarus L (1974) Suppression of human growth hormone secretion by melatonin and cyproheptadine. J Clin invest 54: 116-121.

10. Fideleff H, Aparicio NJ, Guitelman A, Debeljuk L, Mancini A, et al. (1976) Effect of melatonin on the basal and stimulated gonadotropin levels in normal men and postmenopausal women. J Clin Endocrinol Metab 42: 1014-1017.

11. Nordlund Ji, Lerner AB (1977) The effects of oral melatonin on skin and on the release of pituitary hormones. J Clin Endocrinol Metab 45: 768-772.

12. Banerjee S, Margulis L (1973) Mitotic arrest by melatonin. Exp Cell Res 78: 314-318.

13. Ibanez-Costa A, Cordoba-Chacon, Gahete MD, Kineman RD, Castano JP (2015) Melatonin regulates somatotrope and iactotrope function through common and distinct signalling pathways in cultured primary pituitary cells from female primates. Enddocrinology 156: 1100-1110.

14. Herer P, Luboshitzky R, Levi M, Shen-Orr Z, Blumenfeld Z, et al. (2000) Long-term melatonin administration does not alter pituitary-gonadal hormone secretion in normal men. Human Reprod 15: 60-65.

15. Chatteerjee S, Banerji TK (1989) Effects of melatonin on the growth of MtT/F4 anterior pituitary tumor: evidence for inhibition of tumor growth dependent upon the time of administration. I Pineal Res 7: 381-391.

16. Yang QH, Xu IN, Xu RK, Pang SE (2007) Antiproliferative effects of melatonin on the growth of rat pituitary prolactin-secreting tumor cells in vitro. J Pineal Res 42: 172-179.

17.Smisi AA, Pasquali D, D’Apuzzo A, Esposito D, Venditto T, et al. (1997) Twenty-four-hour melatonin patter in acromegaly: effect of acute octreotide administration. Endocrinal Invest 20: 128-133.

18. Quay WB (1967) Spontaneous adenomata of the hypophyseal anterior lobe in aged pinealectomized rat. Experientia 23: 129-130.

19. Regelson W, Pierpaoli W (1987) Melatonin: a rediscovered antitumor hormone? Cancer Invest 5: 379-385.

20. Lissoni P, Mainini E, Rovelli F, Mazzi C, Ardizzoia A, et al. (1992) A clinical study of the pineal hormone melatonin in patients with growth hormone or prolactin-secreting pituitary tumours. Eur J Med 1: 407410 .

21. Terzolo M, Piovesan A, Alì A, Codegone A, Pia A, et al. (1995) Circadian profile of serum melatonin in patients with Cushing's syndrome or acromegaly. J Endocrinol lnvest 18: 17-24.

22. Barath P, Csaba G (1974) Histological changes in the lung, thymus and adrenal one and half year after pinelectomy. Acta Biol Acad Sci Hung 25: $123-128$.

23. Johnson JE (1980) Fine structural effects of hypophysectomy on the aging rat pineal gland. Gerontology 26: 206-216.

24. Lissoni P, Resentini M, Mauri R, De Medici C, Morabito F, et al. (1986) Effect of an acute injection of melatonin on the basal secretion of hypophyseal hormones in prepubertal and pubertal healthy subjects. Acta Endocrinol 111: 305-311.
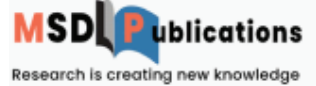
Citation: Lissoni P, Pasquetti M, Pensato S, Messina G, Porro G, et al. (2019) A Clinical Study of Pituitary and Pineal Gland Interactions: Effects of Treatment with The Pineal Hormone Melatonin in Patients with Hypophyseal Adenomas. Rea Int Journal of Endocrinology and Diabetes. 1(1): 006-010. DOI: 10.37179/rijed.000002.

25. Anten Tay F, Chou C, Anton S, Wurtman RY (1968) Brain serotonin concentration following intraperitoneal administration of melatonin. Science 162: 277-280.

26. Lissoni P (1999) The pineal gland as a central regulator of cytokine network. Neuroendocrinol Lett 20: 343-349.

27. Brzezinski A (1997) Melatonin in humans. N Engl J Med 336: 186-195.

28. Chuchuen U, Ebadi M, Govitrapong P (2004) The stimulatory effect of mu- and delta-opioid receptors on bovine pinealocyte melatonin synthesis. J Pineal Res 37: 223-239.

29. Grotenhermen F (2004) Pharmacology of cannabinoids. Neuroendocrinol Lett 25: 14-23.
30. Lissoni P (2014) Melatonin in human cancer: therapeutic possibilities. In Springer India: 43-56

31. Srinivasan V, Brzezinski A, Oter S, Samuel D. Shillcutt (2014) Melatonin and Melatoninergic Drugs in Clinical Practice.

32. Reiter RJ (2004) Mechanisms of cancer inhibition by melatonin. J Pineal Res 37: 213-214.

33. Li Y, Zhou Y, Meng X, Zhang JJ, Xu DP, et al. (2017) Melatonin for the prevention and treatment of cancer. Oncotarget 8: 39896-39921.

34.34. Di Fede G, Messina G, Monzon A, Meli O, Gavazzeni C, et al. (2017) Clinical effects of the pineal antitumor and psychedelic beta-carboline pinealine in the treatment of untreatable metastatic cancer patients. Integr Canc Biol Res 1: 2-4. 